\title{
Localization of an Absorbing Inhomogeneity in a Scattering Medium in a Statistical Framework
}

\author{
Guangzhi Cao, Vaibhav Gaind, Charles A. Bouman, and Kevin J. Webb \\ School of Electrical and Computer Engineering, Purdue University, West Lafayette, Indiana \\ 47907-2314, USA \\ webb@purdue.edu
}

\begin{abstract}
An approach for the fast localization and detection of an absorbing inhomogeneity in a tissue-like scattering medium is presented. The probability of detection as a function of the size, location, and absorptive properties of the inhomogeneity is investigated. The detection sensitivity in relation to the source and detector location serves a basis for instrument design. (c) 2007 Optical Society of America
\end{abstract}

OCIS codes: $170.3010,290.7050,100.3010,100.3190$

Optical imaging in scattering media provides important opportunities for clinical imaging and environmental sensing, among others [1]. In the near-infrared wavelength range, soft tissue has both high scatter and low absorption, allowing use of a diffusion equation model for photon transport $[2,3]$, which with $\exp (j \omega t)$ time dependence is

$$
\left[\nabla \cdot \mathrm{D}(\mathbf{r}) \nabla-\mu_{a}(\mathbf{r})+\frac{j \omega}{c}\right] \phi(\mathbf{r}, \omega)=-\beta \delta\left(\mathbf{r}-\mathbf{r}_{s}\right),
$$

where $\phi$ is the photon flux density, $\omega$ is the circular modulation frequency, $\beta$ is the modulation amplitude, $c$ is the speed of light in the intervening medium between the scatterers, $\mu_{a}$ is the absorption coefficient, $D$ is the diffusion coefficient, and a Dirac delta function excitation is assumed. Reconstruction of the unknown optical parameters $\mu_{a}(\mathbf{r})$ and $D(\mathbf{r})$ requires inversion of measured data, which is formulated as an optimization problem $[1,4]$. This is a computationally intensive process, in large part due to the nonlinear relationship between the cost function and the image parameters. Another difficulty is caused by physical limitations of a practical measurement system, which may result in insufficient information for accurate volumetric imaging. These issues motivate interest in simpler, efficient approaches for detecting and localizing a heterogeneity in a scattering medium instead of quantitative three-dimensional reconstruction.

Methods have been studied for localizing injected fluorophores. Chen et al. used least squares curve fitting to compare a diffusion equation model for expected fluorescence with measurements based on the perturbation on a cancellation plane, formed by dual-interfering sources [5], to localize a fluorophore in a mouse model [6]. Gannot et al. used the Levenberg-Marquardt method to fit measured data with a forward model based on random-walk theory for three-dimensional 
localization of a fluorophore in a mouse tongue [7]. Milstein et al. developed a statistical approach based on maximum likelihood (ML) estimation for localization and a binary hypothesis test to detect a fluorescent source [8]. In this Letter, we extend Milstein's work for fluorescence detection to study issues related to the three-dimensional localization and detection of an intrinsic absorbing inhomogeneity in a scattering medium such as tissue. The probability of detection is used to characterize the diagnostic capability of such a measurement system, and the detection sensitivity presented can be used to optimize the source-detector (SD) geometry, thereby providing a path to instrument design. We also investigate how factors such as SD geometry, and the physical and optical properties of the inhomogeneity, affect detection and localization.

We use ML estimation to estimate the location of an absorbing inhomogeneity in a homogeneous background having parameters $\mu_{a 0}$ and $D_{0}$, which are assumed known. A model is needed to parameterize the unknown inhomogeneity, which could have varying size and optical contrast (defined as $\left.\Delta \mu_{a}=\mu_{a}-\mu_{a 0}\right)$. We use the point inhomogeneity model suggested by Milstein et al. [8], which proved effective in localizing fluorescence, and account for the contrast through a weighting factor for this point absorber, given by $\delta u(\mathbf{r})$. A measurement vector $\mathbf{y}$ of length $M$, for example, the optical intensity at a series of points on the surface at a particular modulation frequency for the light, is compared with a predicted measurement $\mathbf{f}(\mathbf{r})$, based on (1), assuming there exists a point inhomogeneity at position $\mathbf{r}$. Let $\mathbf{y}_{0}$ represent the expected measurement in the absence of an inhomogeneity and $\mathbf{f}^{\prime}(\mathbf{r})$ be the Fréchet derivative which relates perturbations in $\mu_{a}(\mathbf{r})$ to the predicted measurement $\mathbf{f}(\mathbf{r})$, i.e., $\mathbf{f}(\mathbf{r}) \approx \mathbf{y}_{0}+\mathbf{f}^{\prime}(\mathbf{r}) \delta u(\mathbf{r})$. The ML localization can thus be formulated as

$$
C(\mathbf{r}, \delta u(\mathbf{r}))=\arg \min _{\mathbf{r}}\left\|\mathbf{y}-\mathbf{y}_{0}-\mathbf{f}^{\prime}(\mathbf{r}) \delta u(\mathbf{r})\right\|_{\Lambda}^{2},
$$

where $C(\mathbf{r}, \delta u(\mathbf{r}))$ is the negative $\log$ likelihood and is treated as a cost function, $\Lambda^{-1}$ is the noise covariance matrix, for which we use a shot noise model [4], and $\|\mathbf{v}\|_{\mathbf{W}}^{2}=\mathbf{v}^{H} \mathbf{W} \mathbf{v}$, with $H$ being the Hermitian transpose. This optimization can be implemented as a two-step procedure in which, for each discretized position $\mathbf{r}$ over the region of interest, $C(\mathbf{r}, \delta u(\mathbf{r}))$ is minimized with respect to $\delta u(\mathbf{r})$, giving the unique (because $C(\mathbf{r}, \delta u(\mathbf{r}))$ is quadratic) closed form estimate

$$
\begin{aligned}
\delta \hat{u}(\mathbf{r}) & =\arg \min _{\delta u} C(\mathbf{r}, \delta u(\mathbf{r})) \\
& =\frac{\operatorname{Re}\left[\left(\mathbf{y}-\mathbf{y}_{0}\right)^{H} \Lambda \mathbf{f}^{\prime}(\mathbf{r})\right]}{\left\|\mathbf{f}^{\prime}(\mathbf{r})\right\|_{\Lambda}^{2}},
\end{aligned}
$$

and then the ML estimate of inhomogeneity location is given by

$$
\hat{\mathbf{r}}=\arg \min _{\mathbf{r}}\left\|\mathbf{y}-\mathbf{y}_{0}-\mathbf{f}^{\prime}(\mathbf{r}) \delta \hat{u}(\mathbf{r})\right\|_{\Lambda}^{2} .
$$

Fig. 1 shows a simulated reflectance measurement geometry with 5 sources and 5 detectors with a separation of $0.5 \mathrm{~cm}$ on the top surface of a semi-infinite medium, giving $M=25 \mathrm{SD}$ measurement pairs. We consider an $8 \mathrm{~cm} \times 8 \mathrm{~cm} \times 8 \mathrm{~cm}$ computational domain that is discretized with a grid size of $1.25 \mathrm{~mm}$. The background has $\mu_{a 0}=0.02 \mathrm{~cm}^{-1}$ and $D_{0}=0.03 \mathrm{~cm}$. An inhomogeneity of diameter $0.625 \mathrm{~cm}$, having $\mu_{a}=0.12 \mathrm{~cm}^{-1}$ and $D=0.03 \mathrm{~cm}$, is present at depth $d=1.5 \mathrm{~cm}$. We 
assume an average signal-to-noise ratio (SNR) of approximately $40 \mathrm{~dB}$ and a modulation frequency of $\omega=2 \pi \times 10^{6} \mathrm{rad} / \mathrm{s}$. An analytic solution of (1), with an extrapolated $\phi=0$ boundary condition to represent the interface between the scattering medium and free space [8], leads to an expression for $\mathbf{f}^{\prime}(\mathbf{r})$. Fig. 2(a) gives a plot of the negative log likelihood, and the estimated centroid of the inhomogeneity is within $2.5 \mathrm{~mm}$ of the true point. This is a promising result, given the simple measurement geometry. Fig. 2(b) shows the reconstruction of $\mu_{a}$ using the same data set. Note that the reconstructed $\mu_{a}$ is not accurate, which is due to the limited data set. We have previously shown that the reconstruction can be made quantitative with more SD pairs and through use of nonlinear optimization methods [4]. The localization approach we present is thus a computationally efficient way of obtaining the position of an inhomogeneity, and anecdotally success appears possible with very limited measurement data.

Determination of the inhomogeneity's presence, or lack thereof, is a detection problem for which we employ binary hypothesis testing. Let the hypothesis $H_{0}$ correspond to the absence of an inhomogeneity and $H_{1, \mathbf{r}}$ to the presence of an inhomogeneity at position $\mathbf{r}$. The probability densities for $\mathbf{y}$ under the two hypotheses are

$$
\begin{aligned}
p\left(\mathbf{y} \mid H_{1, \mathbf{r}}\right) & =\frac{|\Lambda|}{(2 \pi)^{M}} \exp \left(-\frac{1}{2}\|\mathbf{y}-\mathbf{f}(\mathbf{r})\|_{\Lambda}^{2}\right) \\
p\left(\mathbf{y} \mid H_{0}\right) & =\frac{|\Lambda|}{(2 \pi)^{M}} \exp \left(-\frac{1}{2}\left\|\mathbf{y}-\mathbf{y}_{0}\right\|_{\Lambda}^{2}\right)
\end{aligned}
$$

The likelihood ratio test (LRT) is

$$
L(\mathbf{y}, \mathbf{r})=\log \frac{p\left(\mathbf{y} \mid H_{1, \mathbf{r}}\right)}{p\left(\mathbf{y} \mid H_{0}\right)}=\operatorname{Re}\left[\mathbf{h}(\mathbf{r})^{H}\left(\mathbf{y}-\mathbf{y}_{0}\right)\right]-c(\mathbf{r})
$$

where $\mathbf{h}(\mathbf{r})^{H}=\Delta \mathbf{y}(\mathbf{r})^{H} \Lambda$ can be viewed as a matching filter, $c(\mathbf{r})=(1 / 2)\|\Delta \mathbf{y}(\mathbf{r})\|_{\Lambda}^{2}$ is a constant for each position $\mathbf{r}$, and $\Delta \mathbf{y}(\mathbf{r})=\mathbf{f}(\mathbf{r})-\mathbf{y}_{0}$. Equation (7) provides the highest probability of detection for a specified false alarm rate. The LRT suggests that if the correlation between $\mathbf{y}-\mathbf{y}_{0}$ and $\mathbf{h}(\mathbf{r})$ is above a certain threshold, then we say an inhomogeneity exists. The decision statistic $q=\operatorname{Re}\left[\mathbf{h}(\mathbf{r})^{H}\left(\mathbf{y}-\mathbf{y}_{0}\right)\right]$ has a normal distribution under the two hypotheses, i.e., $\left(q \mid H_{0}\right) \sim N\left(0, \sigma_{q}^{2}\right)$ and $\left(q \mid H_{1, \mathbf{r}}\right) \sim N\left(\bar{q}, \sigma_{q}^{2}\right)$, where both the mean $\bar{q}$ and variance $\sigma_{q}^{2}$ are equal to $\|\Delta \mathbf{y}(\mathbf{r})\|_{\Lambda}^{2}$. For a specified false alarm rate $P_{F}$, the threshold $k_{P_{F}}$ can be determined as $k_{P_{F}}=\sigma_{q} \Phi^{-1}\left(1-P_{F}\right)$, where $\Phi$ is a normal distribution function with mean 0 and variance 1 . Thus we declare that an inhomogeneity exists if $q>k_{P_{F}}$. For a specific measurement system, the probability of detection is

$$
P_{D}=\int_{k_{P_{F}}}^{\infty} p\left(q \mid H_{1, \mathbf{r}}\right) d q=1-\Phi\left(\frac{k_{P_{F}}-\bar{q}}{\sigma_{q}}\right) .
$$

Consider now the influence of physical (size, depth) and optical (contrast) properties of the inhomogeneity on $P_{D}$, assuming that these properties are known. In practice, the parameters describing the inhomogeneity are unknown and must be estimated. Therefore, the results of our simulation, with $P_{D}$ computed using (8), gives an upper bound for the $P_{D}$ of a measurement system. The measurement geometry of Fig. 1 is used. Fig. 3(a) plots $P_{D}$ as a function of the inhomogeneity 
depth for the case of Fig. 2. $P_{D}$ decreases as the inhomogeneity depth increases, and the reliable detection depth is about $2 \mathrm{~cm}$. Fig. $3(\mathrm{~b})$ gives $P_{D}$ as a function of inhomogeneity size and contrast for a fixed depth of $1.5 \mathrm{~cm}$. Notice that detection becomes more reliable as both the size and contrast increase, with a fixed source power (SNR). Fig. 3(c) shows $P_{D}$ as a function of inhomogeneity depth and contrast. The achievable detection depth increases with the contrast but finally saturates at about $3 \mathrm{~cm}$. This saturation is dictated by the detector noise, i.e., by the SNR. Fig. 3(d) gives $P_{D}$ as a function of inhomogeneity depth and size. The achievable detection depth increases with the inhomogeneity size, but saturates also due to the noise floor.

The placement and number of sources and detectors amounts to instrument design. Our strategy is to maximize the detection sensitivity $S=\left|y_{i}-y_{0 i}\right|^{2} / y_{0 i}$ for each SD pair, where $y_{i}$ is the element of $\mathbf{y}$ with $\mathrm{S}_{i}-\mathrm{D}_{i}$ and the inhomogeneity present, and $y_{0 i}$ that without the inhomogeneity. An increase in $S$ corresponds to an increase in $P_{D}$, as (8) indicates. The analytical result for the sensitivity is plotted in Fig. 4 for inhomogeneity depths of $2 \mathrm{~cm}$ and $3 \mathrm{~cm}$. The optimal SD separations are about $2.3 \mathrm{~cm}$ and $3.5 \mathrm{~cm}$, respectively. The optimal SD separation increases as the inhomogeneity depth increases, ultimately being limited by the detector noise floor. By obtaining such information, one can optimize the design of a measurement system. A convenient approximation for the closed form semi-infinite medium solution for $S$ can be found under the assumption that $d>>l^{*}$ and $\gamma>>l^{*}$, where $l^{*}=3 D$ is the mean free path and $\gamma$ is the distance between the SD pair, which we find to be

$$
S \approx A \cdot \frac{\gamma^{2} \exp \left\{-4 k\left(\frac{\gamma^{2}}{4}+d^{2}\right)^{1 / 2}\right\}}{\left(\frac{\gamma^{2}}{4}+d^{2}\right)^{4} \exp \{-k \gamma\}},
$$

where $A$ is a constant and $k=\left[\left(c^{2} \mu_{a}^{2}+\omega^{2}\right) /\left(c^{2} D^{2}\right)\right]^{1 / 4} \cos \left[(1 / 2) \tan ^{-1}\left(\omega /\left(c \mu_{a}\right)\right)\right]$ is the decay coefficient. The scaled result from (9) is shown as points in Fig. 4, and these agree nicely with the analytical result. An instrument should have SD spacings that encompass the optimum sensitivity, which for the two cases we consider are given by the peaks in Fig. 4.

We presented a statistical framework for fast localization and detection of an absorbing inhomogeneity in a scattering medium. Extension to a diffusion coefficient inhomogeneity would follow the same general procedure. With a known inhomogeneous background, the forward model could be calculated numerically, and the Fréchet matrix elements stored beforehand.

We acknowledge funding from the National Science Foundation under award CCR-0431024.

\section{References}

1. A. P. Gibson, J. C. Hebden, and S. R. Arridge, "Recent advances in diffuse optical imaging," Phys. Med. Biol 50, R1-R43 (2005).

2. M. S. Patterson, J. D. Moultan, B. C. Wilson, K. W. Berndtand, and J. R. Lakowicz, "Frequency-domain reflectance for the determination of the scattering and absorption properties of tissue," Appl. Opt. 30, 4474-4476 (1991).

3. M. A. O'Leary, D. A. Boas, B. Chance, and A. G. Yodh, "Refraction of diffuse photon density waves," Phys. Rev. Lett. 69, 2658-2661 (1992). 
4. A. B. Milstein, S. Oh, J. S. Reynolds, K. J. Webb, C. A. Bouman, and R. P. Millane, "Threedimensional Bayesian optical diffusion tomography with experimental data," Opt. Lett. 27, 95-97 (2002).

5. M. G. Erickson, J. S. Reynolds, and K. J. Webb, "Comparison of sensitivity for single and dual interfering source configurations in optical diffusion imaging," J. Opt. Soc. Am. A 14, 3080-3092 (1997).

6. Y. Chen, G. Zheng, Z. H. Zhang, D. Blessington, M. Zhang, H. Li, Q. Liu, L. Zhou, X. Intes, S. Achilefu, and B. Chance, "Metabolism-enhanced tumor localization by fluorescence imaging: in vivo animal studies," Opt. Lett. 28, 2070-2072 (2003).

7. I. Gannot, A. Garashi, G. Gannot, V. Chernomordik, and A. Gandjbakhche, "In vivo quantitative three-dimensional localization of tumor labeled with exogenous specific fluorescence markers," Appl. Opt. 42, 3073-3080 (2003).

8. A. B. Milstein, M. D. Kennedy, P. S. Low, C. A. Bouman, and K. J. Webb, "Statistical approach for detection and localization of a fluorescing mouse tumor in a turbid medium," Appl. Opt. 44, 2300-2310 (2005). 


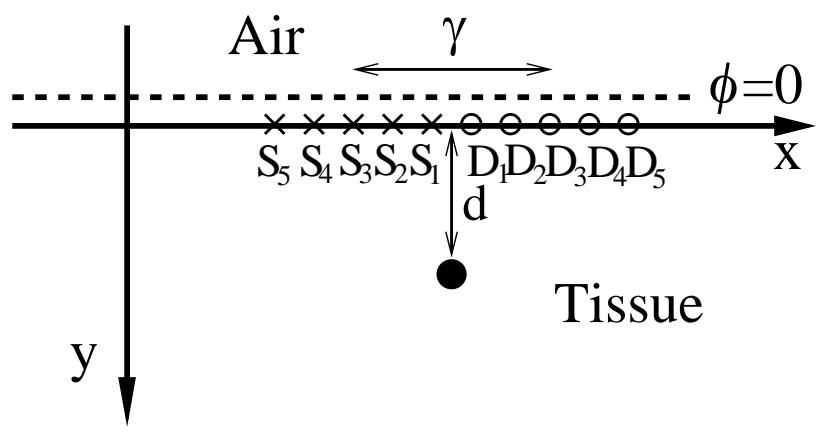

Fig. 1. Measurement geometry for localization. A spherical absorber at depth $d$ is assumed in the simulation. The background optical parameters are: $\mu_{a 0}=0.02 \mathrm{~cm}^{-1}$, $D_{0}=0.03 \mathrm{~cm}$, and the modulation frequency is $\omega=2 \pi \times 10^{6} \mathrm{rad} / \mathrm{s}$.

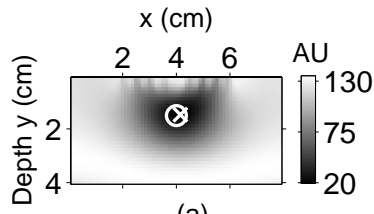

(a)

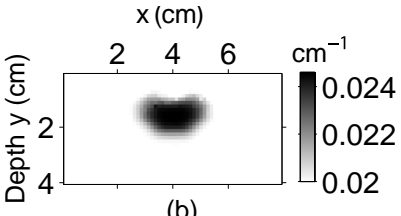

(b)

Fig. 2. Localization versus reconstruction: (a) Negative log likelihood: o denotes the true inhomogeneity location and $\times$ the estimated location. (b) Optical diffusion tomography reconstruction of $\mu_{a}$. Parameters: 5 sources and 5 detectors and background parameters as in Fig. 1; inhomogeneity $\mu_{a}=0.12 \mathrm{~cm}^{-1}, D=0.03 \mathrm{~cm}$; average SNR is $40 \mathrm{~dB}$; spherical inhomogeneity diameter of $0.625 \mathrm{~cm}$. 


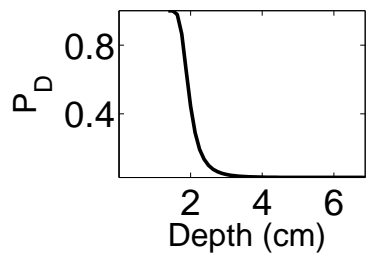

(a)

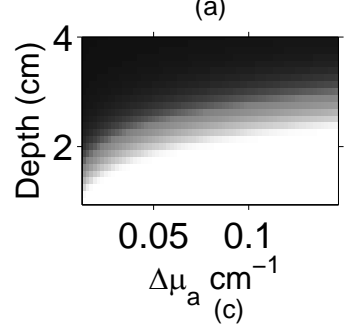

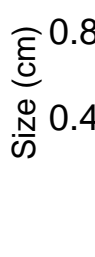

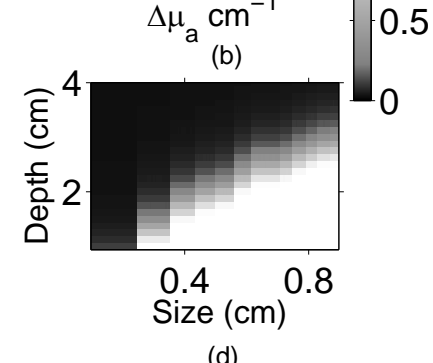

Fig. 3. Influence of inhomogeneity depth, size and optical contrast $\left(\Delta \mu_{a}\right)$ on $P_{D}$ for the geometry and parameters shown in Fig. 1, with $P_{F}=0.03$ and an average SNR of $40 \mathrm{~dB}$. (a) $P_{D}$ as a function of depth, for an inhomogeneity having: diameter $0.625 \mathrm{~cm}, \mu_{a}=0.12 \mathrm{~cm}^{-1}$, and $D=0.03 \mathrm{~cm}$. (b) $P_{D}$ as a function of size and $\Delta \mu_{a}$, with $d=1.5 \mathrm{~cm}$. (c) $P_{D}$ as a function of depth and $\Delta \mu_{a}$, with a $0.625 \mathrm{~cm}$ diameter inhomogeneity. (d) $P_{D}$ as a function of depth and size, with $\Delta \mu_{a}=0.1 \mathrm{~cm}^{-1}$.

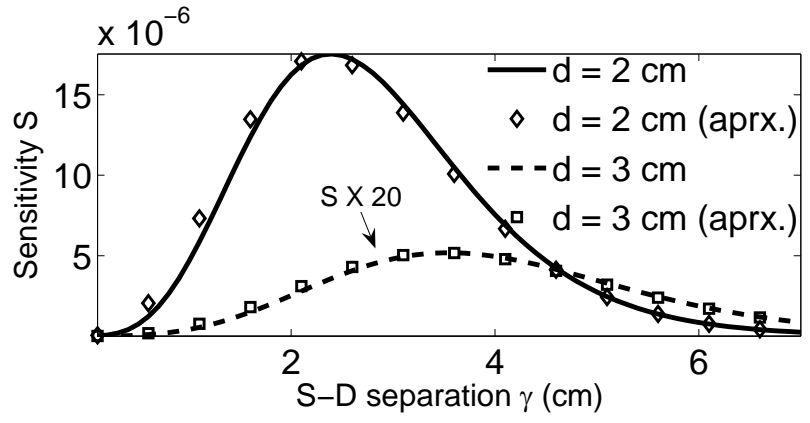

Fig. 4. Detection sensitivity as a function of S-D distance for two inhomogeneity depths. The background optical parameters are: $\mu_{a 0}=0.1 \mathrm{~cm}^{-1}, D_{0}=0.03 \mathrm{~cm}$, which give $k=0.9 \mathrm{~cm}^{-1}$. The sensitivity for inhomogeneity depth $3 \mathrm{~cm}$ is magnified 20 times. The points are the approximate solution from (9). 